\title{
Comparing and unifying slope estimates across psychometric function models
}

\author{
JAMES M. GILCHRIST, DAVID JERWOOD, and H. SAM ISMAIEL \\ University of Bradford, Bradford, England
}

\begin{abstract}
The psychometric function relating stimulus intensity to response probability generally presents itself as a monotonically increasing sigmoid profile. Two summary parameters of the function are particularly important as measures of perceptual performance: the threshold parameter, which defines the location of the function over the stimulus axis (abscissa), and the slope parameter, which defines the (local) rate at which response probability increases with increasing stimulus intensity. In practice, the psychometric function may be modeled by a variety of mathematical structures, and the resulting algebraic expression describing the slope parameter may vary considerably between different functions fitted to the same experimental data. This variation often restricts comparisons between studies that select different functions and compromises the general interpretation of slope values. This article reviews the general characteristics of psychometric function models, discusses three strategies for resolving the issue of slope value differences, and presents mathematical expressions for implementing each strategy.
\end{abstract}

The psychometric function, which describes how the response of an observer on a psychophysical task depends on some physical stimulus value, is of fundamental importance in perception and psychophysics. For example, the ability of an observer to detect a visual stimulus depends on the contrast of that stimulus, and this dependence is described by the psychometric function for contrast detection (see, e.g., Nachmias, 1981). Sensory responses in such tasks are typically expressed either as probabilities (hit rate or proportion correct) or in terms of effect size measures such as $d^{\prime}$, defined in signal detection theory as the standardized difference between the expected intensities of pure noise and noisy signal distributions (Green $\&$ Swets, 1966; Harvey, 1986). When the response is expressed as $d^{\prime}$, psychometric functions are often described as power functions (Pelli, 1987), but when it is expressed as a probability, psychometric functions are necessarily continuous and monotonically increasing against the stimulus value according to some sigmoid profile (see Figure 1).

The structure of any psychometric probability function can be fully described by four operating parameters (Treutwein, 1995; Treutwein \& Strasburger, 1999). One parameter defines the location of the function on the abscissa, a second defines its local gradient (slope) at some specific location, and the others define the horizontal asymptotes (see Figure 1).

The authors are grateful to Bernhard Treutwein and two anonymous reviewers for detailed, constructive comments on an earlier version of the manuscript. Correspondence concerning this article should be addressed to J. M. Gilchrist, Department of Optometry, University of Bradford, Richmond Road, Bradford BD7 1DP, England (e-mail: j.m.gilchrist@ bradford.ac.uk).
An important class of psychometric functions covers those that describe probabilities of a correct response in a forced choice task or of the detection of the stimulus in a yes/no task. The asymptotic parameters then are probabilities determining the limits of sensory performance. The lower asymptote, which denotes the probability of a correct guess, will be the false alarm rate when subjects give yes/no responses, or $1 / m$ for $m$-alternative forced choice responses. The upper asymptote marks the limit of accuracy of the perceptual process and will usually exceed .95 . The shortfall between this probability limit and certainty (a probability of 1.0) denotes the lapsing rate of the subject, which is the probability that even a strong stimulus may be overlooked because of fatigue or lack of concentration.

The location parameter has the same units as the stimulus; it is generally interpreted as a measure of sensory threshold, and its reciprocal as a measure of sensitivity. Subsequently, we will refer to this as the threshold parameter. The slope parameter, which often represents the gradient at the threshold location, determines the rate of change in response probability per unit change in stimulus level. The reciprocal of the slope parameter value is a measure of the spread of the function on the stimulus axis. The threshold and slope parameters are of particular importance in defining the pragmatic stimulus-response relationship.

When psychometric functions are fit to experimental data, it is possible to estimate all four parameters (Treutwein \& Strasburger, 1999), but threshold estimation has generally dominated experimental psychophysics, to such an extent that other parameters have been either ignored or assumed to have constant values (Treutwein, 1995). There is, however, a growing awareness that it 


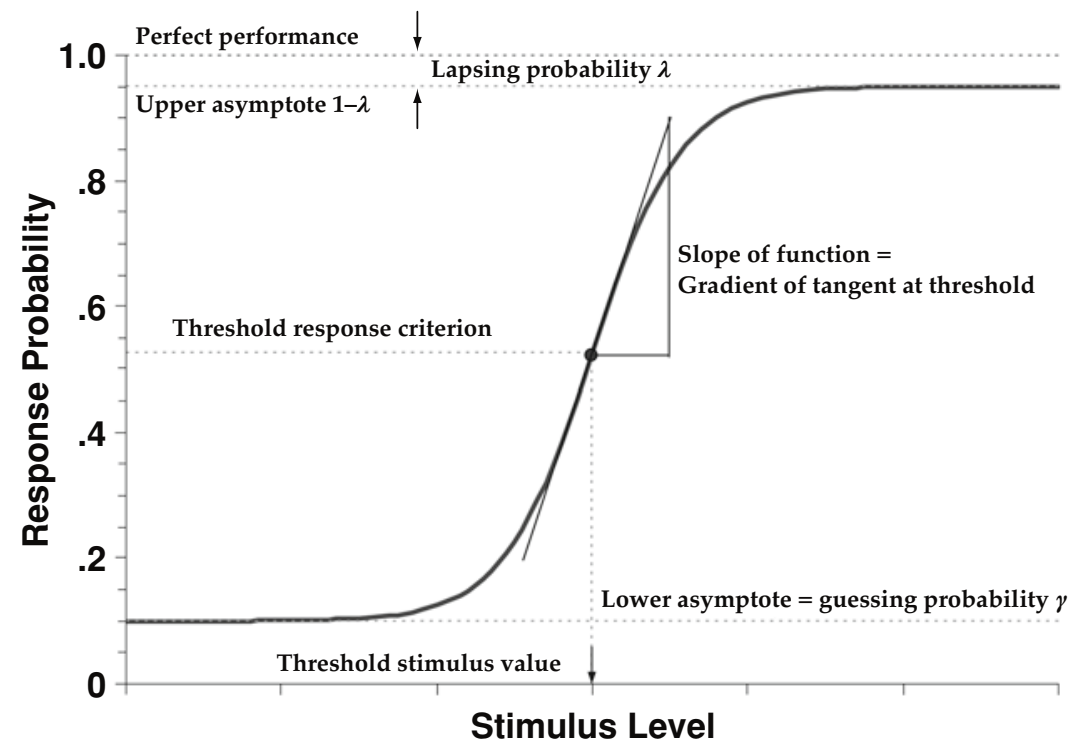

Figure 1. The psychometric function relating response probability to stimulus intensity. Abscissa: stimulus level increasing from left to right on an arbitrary scale, which may be arithmetic (linear) or logarithmic. Ordinate: response probability. Lower asymptote: probability of correct guess $\gamma$ (false positive response). Upper asymptote: $1-$ probability of lapse $\lambda$ (false negative response). Threshold criterion: a specified response probability corresponding to a particular threshold stimulus value. Slope of the function: local gradient of the function at the threshold point. See text for details.

may also be beneficial to estimate asymptotic parameters (Wichmann \& Hill, 2001), and in particular, the importance of the slope parameter as a measure of perceptual performance and clinical status is now acknowledged (Chauhan, Tompkins, LeBlanc, \& McCormick, 1993; Patterson, Foster, \& Heron, 1980; Strasburger, 2001b; Tyler, 1997). Consequently, recently developed adaptive procedures deliver estimates of both threshold and slope (Kaernbach, 2001; King-Smith \& Rose, 1997; Kontsevich \& Tyler, 1999; Snoeren \& Puts, 1997).

Estimation of psychometric function parameters is usually carried out under the assumption of a specific mathematical model of the function. Although all psychometric probability functions have the same general structure (see Figure 1), different mathematical models are employed in practice, and parameter estimates for different functions fitted to the same data will vary depending on the model adopted. For the two asymptotic parameters and threshold, this is generally not a significant problem, since the range of variation in estimated values is likely to be relatively small, and interpretation of these parameter values is unambiguous; the asymptotes are response probabilities, and the threshold stimulus value can always be defined in terms of some associated response probability. The value of the slope parameter, on the other hand, may vary considerably when different function models are fitted, and the slope value does not have any fixed framework (units or range of values) for its interpretation. For example, Figure 2 shows hypothetical psychometric functions for contrast detection fitted using different models, the gradients of which are matched over the central part of the curve. For all the models, threshold contrast is 0.04 $(\log$ contrast $=-1.40)$. In Figure $2 \mathrm{~A}$, plotted on a logarithmic stimulus scale, Models 1 and 2 are matched with slope parameter values of 3.50 and 11.17 , respectively. In Figure 2B, plotted on an arithmetic stimulus scale, the same Model 1 with a slope parameter value of 3.50 is now matched by Model 3 with a value of 121.30 .

The occurrence of such marked variation in the value of the slope parameter, on moving from one psychometric function model and/or stimulus scale to another, limits researchers' ability to compare results from different studies and, therefore, compromises the general interpretation of slope values. It would be helpful to have general awareness and, perhaps, agreement on strategies for resolving slope value differences, so that results from experiments using different functions might be compared without the need for access to the original data. In a clinical context, the ability to convert slope values could facilitate achieving a single measure of psychometric slope by which the status of patients could be quantified unambiguously.

Strasburger (2001a) has presented expressions for converting slope parameter values between psychometric functions, using the principle of matching functions at the point of maximum slope. The present article seeks to extend the analysis to include aspects that have not been addressed previously. Although many of these are widely recognized by those experienced in this field, there appears to be no literature that presents an overview. We therefore will begin by elucidating some important gen- 

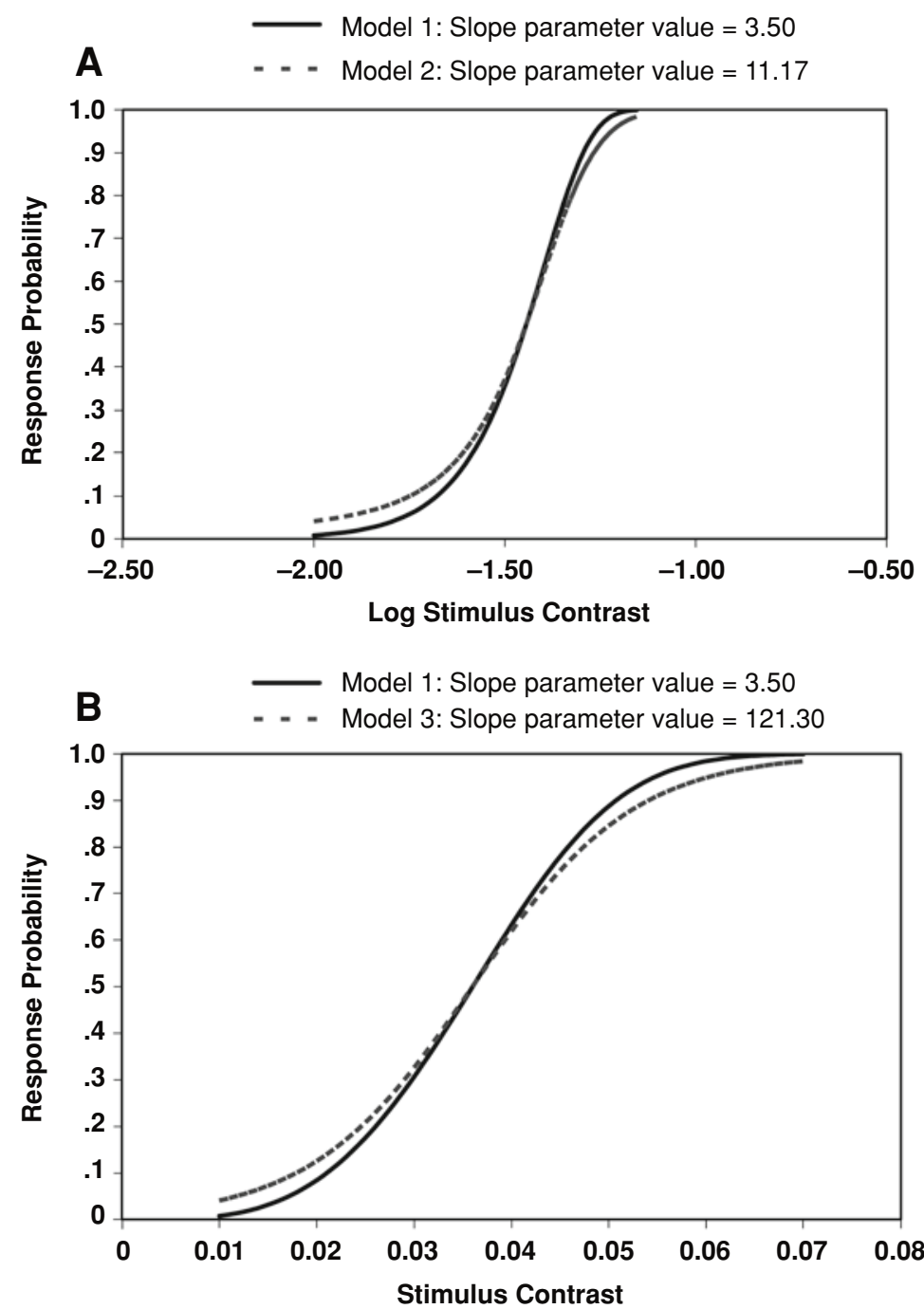

Figure 2. Slope parameter values in different psychometric function models. Functions represent modeled responses from a hypothetical contrast detection experiment with a subject having a median threshold contrast of 0.04 . (A) Stimulus contrast is expressed on a logarithmic $\left(\log _{10}\right)$ scale, and Psychometric Function Models 1 and 2 are matched over the central region, with slope values of 3.50 and 11.17, respectively. (B) Stimulus contrast is expressed on an arithmetic scale, and Psychometric Function Models 1 and 3 are matched over the central region, with slope values of 3.50 and 121.30 .

eral characteristics of psychometric functions and then will define the principal psychometric function models, discuss three strategies for resolving slope parameter differences, and present all the mathematical expressions required to implement these.

\section{General Characteristics of Psychometric Functions}

Some structural characteristics of psychometric functions affect the general approach to analysis. These concern (1) commonly used models of the psychometric function, (2) forms of the function kernel, (3) logarithmic transformation of the function, (4) independent effects of location and slope parameters, and (5) the effects of asymptotic parameters.

Common models. Psychometric functions are generally modeled using existing statistical (cumulative) distribution functions. Three forms occur most frequently: Gaussian, also referred to as Gaussian integral, cumulative Gaussian, or cumulative normal (Blackwell, 1953; Green \& Swets, 1966; Laming, 1986); logistic (Berkson, 1951; Macmillan \& Creelman, 1991); and Weibull (Quick, 1974; Watson, 1979; Weibull, 1951). Authors sometimes attempt theoretical justification for the distribution adopted, 
but often the choice is a matter of convenience, since all three models adopt structurally similar shapes (Harvey, 1986).

Function kernels. The three most popular psychometric functions are defined by different mathematical expressions, but all have similar kernels - the kernel being that part at the heart of the expression that combines the stimulus value with the two functional parameters. Two fundamental kernel forms are apparent, which are based either on scaled differences of the form

$$
v_{3}\left(v_{1}-v_{2}\right) \quad \text { (difference kernel) }
$$

or on powered ratios of the form

$$
\left(\frac{v_{1}}{v_{2}}\right)^{v_{3}} \quad \text { (ratio kernel), }
$$

where $v_{3}$ always represents the slope parameter, $v_{1}$ represents a variable stimulus value, and $v_{2}$ represents a fixed threshold parameter, or vice versa, depending on the particular model in which the kernel appears. The next two discussion points will explain why both kernel forms are necessary.

Logarithmic transformation. Sensory stimuli are sometimes scaled arithmetically and sometimes logarithmically, so psychometric functions that can accommodate both formulations must be available. The following text will generally refer to these as functions in $x$ and functions in $\log x$, respectively. If the function in $x$ uses the difference kernel, logarithmic transformation is impossible, because there will always be some range of stimulus values $(x)$ over which the kernel is less than or equal to zero. Over such domains, the logarithm function is not defined. This will occur at threshold $\left(v_{1}=v_{2}\right)$ and either above threshold, if the threshold is represented by $v_{1}$ and the stimulus value by $v_{2}$, or below threshold, if the threshold is $v_{2}$ and the stimulus value is $v_{1}$. The ratio kernel, however, naturally permits logarithmic transformation, to give

$$
v_{3}\left(\log v_{1}-\log v_{2}\right) \quad \text { (log-ratio kernel). }
$$

Using the ratio kernel and its logarithmic equivalent enables a psychometric function to be transformed to give identical response probabilities for a particular stimulus, be it measured on an arithmetic or a logarithmic scale. The logarithmic transformation of the ratio kernel is structurally equivalent to the difference kernel, but with both stimulus and threshold values registered on a logarithmic scale.

Independence of threshold and slope parameters. Since the ratio kernel permits logarithmic transformation, whereas the difference kernel does not, it would seem that ratio kernel forms of the psychometric function are preferable and that the difference kernel offers no advantage. However, a practical limitation of functions using a ratio kernel on arithmetic scales is that the effects of threshold and slope parameters are not algebraically independent. That is, changing the threshold parameter value will also change the steepness of the plotted function at that loca- tion (Figure 3A). Consequently, functions sharing a common value of $v_{3}$ but having different threshold values will not appear parallel when plotted. The difference kernel and log-ratio kernel, on the other hand, behave as though location and slope parameters have algebraically independent effects (Figure 3B). If this is considered desirable in practical contexts, it is necessary to use a difference kernel when the stimulus scale is arithmetic and a log-ratio kernel when it is logarithmic. For example, most parametric adaptive methods for estimating function thresholds and slopes require that model functions run parallel for different values of the threshold parameter (Treutwein, 1995; Watson \& Pelli, 1983).

Effects of asymptotic parameters. The analyses to follow will concentrate principally on strategies for resolving differences between slopes of different functions, with the assumption that the upper (lapsing) and lower (guessing) asymptotes are fixed theoretically. However, the asymptotic values affect the analysis by determining the probability range covered by the function, so they must be taken into account. Fortunately, their effects may be considered in terms of a linear transformation of probability values, with the psychometric function commonly written (Klein, 2001; Treutwein, 1995) as follows:

$$
P^{*}(x)=\gamma+(1-\gamma-\lambda) \cdot P(x),
$$

where $\gamma$ is the guessing probability (lower asymptote), $\lambda$ is the lapsing probability (upper asymptote), $P(x)$ is the psychometric function that goes from probability 0 to 1 , and $P^{*}(x)$ is the psychometric function that goes from probability $\gamma$ to $(1-\lambda)$ (see Figure 1$)$.

For the remainder of this article, it will be assumed that all psychometric functions are defined as $P(x)$ above, so conversions between functions will always assume probabilities in the range 0 to 1 (i.e., $\gamma=\lambda=0$ ). If parameter conversion is desired from some originating function to some target function when $\gamma>0$ and/or $\lambda>0$, Equation 3B (inverse of 3A) is used at the outset to transform the originating function from $P^{*}(x)$ to $P(x)$ thus:

$$
P(x)=\frac{P^{*}(x)-\gamma}{1-\gamma-\lambda} .
$$

Following this, the slope conversion is applied, and then Equation $3 \mathrm{~A}$ is used to transform the target function from $P(x)$ to $P^{*}(x)$. This approach is mathematically consistent and simplifies the expressions for slope transformation that will be introduced later.

\section{Psychometric Function Model Specifications}

Gaussian models. The Gaussian (or normal) distribution function, G1x, takes the form

$$
P_{\mathrm{G} 1 x}=\frac{\alpha}{\sqrt{2 \pi}} \int_{-\infty}^{x} \exp \left[-\frac{1}{2}\{\alpha(t-\theta)\}^{2}\right] d t .
$$

This is in difference kernel form; $\theta$ is the threshold parameter located at the median of the distribution, and $\alpha$ is the slope parameter $(1 / S D)$. The Gaussian function is antisymmetric about its median, which is the point of 

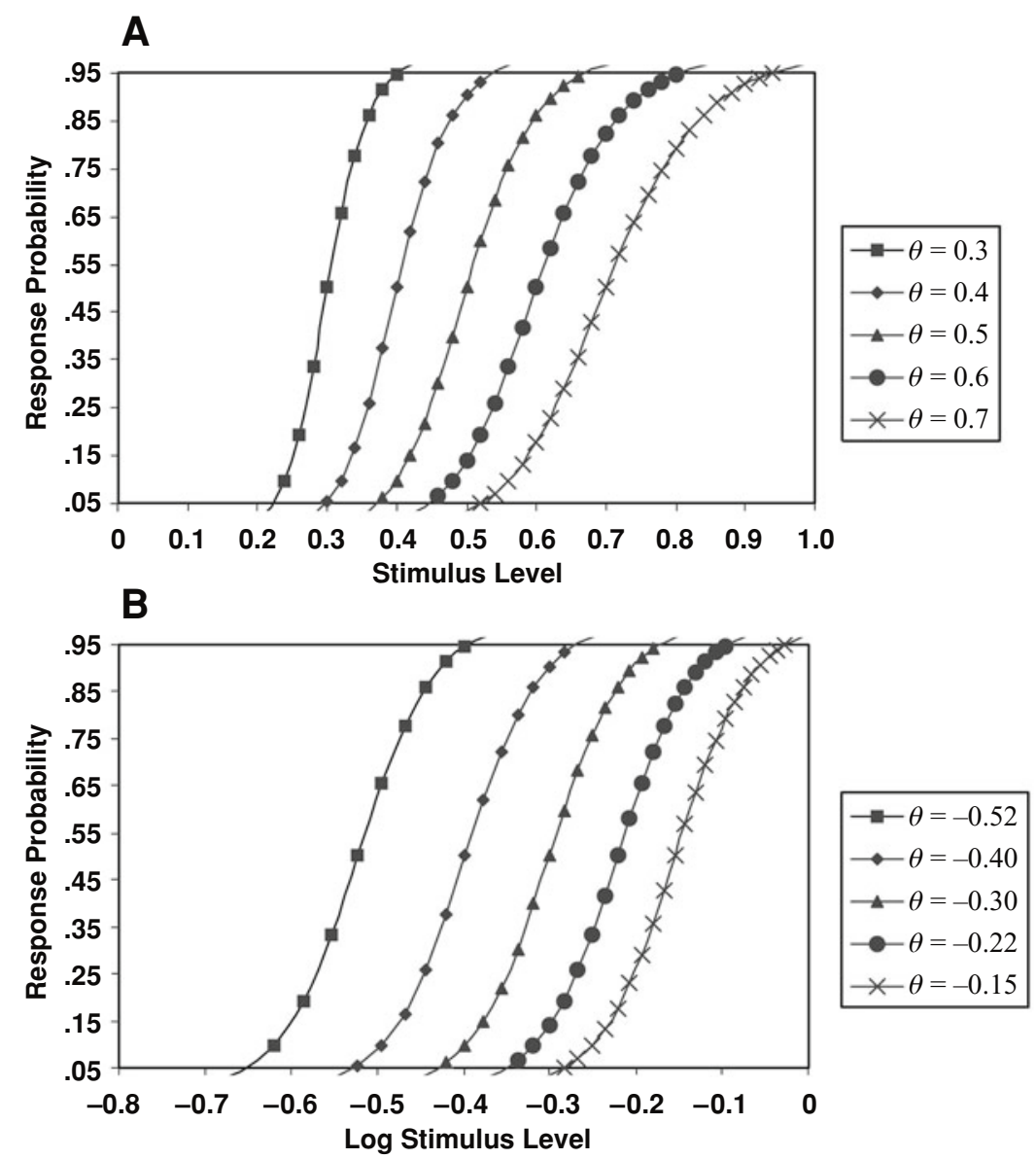

Figure 3. Lack of algebraic independence of threshold and slope parameters in ratio kernel functions on arithmetic stimulus scales. (A) Functions having a constant slope parameter value but a variable threshold parameter $\theta$ do not appear parallel when plotted on an arithmetic stimulus scale. (B) The same functions plotted on a logarithmic stimulus scale appear parallel, demonstrating independence of threshold and slope parameters.

maximum slope, and the response probability at this location is $P_{\mathrm{G} 1 x}(\theta)=.5$. It is, therefore, convenient to refer to parameter $\theta$ as the median threshold parameter, meaning that it defines the stimulus value corresponding to the $50 \%$ (median) point of the response probability scale.

Function G1 $x$ is unsuitable for logarithmic transformation, as has been discussed previously. A logarithmic version of the Gaussian (lognormal), G2x, written in ratio kernel form, is given (Evans, Hastings, \& Peacock, 1993) as

$$
P_{\mathrm{G} 2 x}=\frac{\beta}{\sqrt{2 \pi} \ln b} \int_{0}^{x} \frac{1}{t} \exp \left[-\frac{1}{2}\left\{\log _{b}\left[\frac{t}{\theta}\right]^{\beta}\right\}^{2}\right] d t,
$$

where $b>1$ is the base of the logarithmic transform. The same function in $\log$-ratio kernel form is $\mathrm{G} 2 \log x$,

$$
\begin{aligned}
P_{\mathrm{G} 2 \log x}= & \frac{\beta}{\sqrt{2 \pi} \ln b} \\
& \cdot \int_{0}^{x} \frac{1}{t} \exp \left[-\frac{1}{2}\left\{\beta\left(\log _{b} t-\log _{b} \theta\right)\right\}^{2}\right] d t .
\end{aligned}
$$

The formulations of the Gaussian function in Equations 5 and 6 above are equivalent, and both necessitate logarithmic transformation of the stimulus scale. Henceforth, this function, written in either form above, will be identified as $\mathrm{G} 2 \log x$. Each G2 formulation has threshold parameter $\theta$, and its slope parameter $\beta$ is equal to $1 / S D$.

The integral nature of the Gaussian distribution function introduces mathematical difficulties, so other functions of similar form are often chosen in preference. One that corresponds closely to the Gaussian in shape is the logistic.

Logistic models. A natural two-parameter form of the logistic for use in psychophysics is L1 $x$,

$$
P_{\mathrm{L} 1 x}=[1+\exp \{\delta(\theta-x)\}]^{-1},
$$

where $x$ is stimulus magnitude, $\delta$ is the slope parameter, and $\theta$ again defines the median threshold, where response probability is $P_{\mathrm{L} 1 x}(\theta)=.5$. As with the Gaussian function, the logistic is antisymmetric about this point. Since Equation 7 is of the difference kernel form, its parameters $\theta$ and $\delta$ provide independent control of threshold and slope, respectively. 
Other forms of this logistic function, equivalent to L1x, are also available. A common alternative, in which the parameters do not give independent control of location and slope, is $P(x)=[1+\exp \{\alpha-b x\}]^{-1}$. This can be obtained from L $1 x$ by substituting $b=\delta$ and $a=\delta \theta$. In addition, sigmoid profiles based on the hyperbolic tangent function have been widely adopted in vision science, at least since Naka and Rushton (1966), in order to fit physiological response functions of visual system neurones (Lipetz, 1969). A hyperbolic tangent function algebraically equivalent to $\mathrm{L} 1 x$ is $P_{\mathrm{L} 1 x}(x)=.5+.5$ $\tanh \{.5 \delta(x-\theta)\}$. Strasburger (2001a) has also presented a formulation of the psychometric function based on the hyperbolic tangent.

Since the logistic L1 $x$ is of difference kernel form, logarithmic transformation is not possible. An alternative in ratio kernel form, $\mathrm{L} 2 x$, is

$$
P_{\mathrm{L} 2 x}=\left[1+\left(\frac{\theta}{x}\right)^{\varepsilon}\right]^{-1},
$$

where slope is given by the parameter $\varepsilon$. On a logarithmic stimulus scale, this gives L2 $\log x$,

$$
P_{\mathrm{L} 2 \log x}=\left[1+\exp \left\{\phi\left(\log _{b} \theta-\log _{b} x\right)\right\}\right]^{-1},
$$

where $b>1$ is the base of the logarithmic transform, $\log _{b} \theta$ is the median threshold value on the logarithmic scale, and $\phi$ is the corresponding slope value.

Weibull models. The two-parameter Weibull function (Weibull, 1951) has proved extremely useful in visual psychophysics since its application by Quick (1974) to a model of contrast detection. The most commonly cited form (Nachmias, 1981; Watson, 1979) of the Weibull, $\mathrm{W} 2 x$, is

$$
P_{\mathrm{W} 2 x}=1-e^{\left[-\left(\frac{x}{\eta}\right)^{\omega}\right]},
$$

where $x$ is stimulus magnitude, and now parameter $\eta$ represents threshold and $\omega$ slope. In this expression, the threshold parameter corresponds to a response probability of $P_{\mathrm{W} 2 x}(\eta)=1-e^{-1}=.632$. Weibull model W2x is widely used in reliability analysis, where parameter $\eta$ is often called the characteristic life of the function. In the present context, therefore, it is convenient to refer to this as the characteristic threshold parameter, in order to distinguish it from the median threshold parameter defined earlier.

Psychometric function W2 $x$ is one of a family of functions having the same shape and taking the general form

$$
P_{x}=1-R^{- \text {kernel }},
$$

where the kernel has the ratio form shown in Equation 10. In all such functions, the response probability at threshold $t$ is given by $P_{x}(t)=1-R^{-1}$; therefore, the value of $R$ may be chosen to ensure that the threshold location will correspond to any desired response probability. Thus, if it is desired to retain the Weibull structure but to relocate the threshold naturally at a response probability of .50 , corresponding to the thresholds of Gaussian and logistic functions, equation W2 $x$ may be modified to the following form (Quick, 1974; Wilson, 1980), henceforth called the Quick function, Q2x:

$$
P_{\mathrm{Q} 2 x}=1-2^{\left[-\left(\frac{x}{\theta}\right)^{\omega}\right]} .
$$

In this formulation, $\theta$ corresponds to the median threshold, as defined previously, since $P_{\mathrm{Q} 2 x}(\theta)=1-2^{-1}=$ .50 .

Logarithmic transformation of Weibull and Quick functions, W2 $\log x$ and Q2 $\log x$, respectively, gives

$$
P_{\mathrm{W} 2 \log x}=1-e^{\left[-b^{\wedge}\left\{\omega\left(\log _{b} x-\log _{b} \eta\right)\right\}\right]}
$$

and

$$
P_{\mathrm{Q} 2 \log x}=1-2^{\left[-b^{\wedge}\left\{\omega\left(\log _{b} x-\log _{b} \theta\right)\right\}\right]},
$$

where $b>1$ is the base value for logarithmic transformation. A notable feature of the Weibull/Quick functions is that, unlike the Gaussian and logistic functions, the slope parameter $\omega$ takes the same value on both arithmetic and logarithmic scales.

The $\log$ Weibull model (W2 $\log x$ ) is also known as the Gumbel model (Evans et al., 1993; Treutwein, 1995). This formulation has the advantage that threshold and slope parameters act independently, so profiles having the same slope value but different threshold locations will appear parallel when plotted on a logarithmic stimulus scale. It has been widely used in vision science, although a variety of different formulations appear in the literature. For example, Watson and Pelli (1983) and Harvey (1986) used Equation 13 with a $\log _{10}$ stimulus scale, whereas Treutwein (1995) showed the same formulation with a $\log _{e}$ stimulus scale.

Another important feature to be considered is that, unlike Gaussian and logistic functions, Weibull/Quick functions are not antisymmetric about their median value, so although the Quick form of the Weibull has its natural threshold at the .5 probability location, this is not the point of maximum gradient. In fact, both Weibull and Quick functions experience maximum gradient at the location defined by the characteristic threshold parameter $\eta$ in the W $2 x$ formulation.

Relationship between Weibull and Quick formulations. Note that the same symbol $\omega$ is used for the slope parameter of both Weibull (W2x) and Quick (Q2x) functions. The two functions can be matched exactly, with the same slope $\omega$, by a threshold transformation. Setting Weibull Equation 10 equal to Quick Equation 12, it can be shown that

$$
\theta=\eta(\ln 2)^{1 / \omega} .
$$

When the same functions are expressed on a logarithmic scale (base $b>0$ ), this matching expression becomes

$$
\log _{b} \theta=\log _{b} \eta+\frac{1}{\omega} \log _{b}(\ln 2) .
$$


Hence, Weibull and Quick functions on logarithmic scales are identical but for a location shift along the stimulus axis from characteristic threshold $\eta$ to median threshold $\theta$ (see also Strasburger, 2001a). The exact equivalence of these two functions with a simple threshold transformation is extremely important in the analysis to be presented later.

Having specified the most popular psychometric functions whose interrelationships need to be determined, the discussion can now move to the principles of resolving differences between slope values. For ease of reference, Table 1 summarizes the functions introduced above and the notation to be used in the remainder of the article.

\section{Resolving Slope Differences}

We now will consider three strategies for resolving slope differences between psychometric functions: (1) transform all slope values to obtain a standardized slope measure that is independent of any function, (2) transform the slope value from any originating function to any desired target function, and (3) express the slope value in terms of the spread of its function on the stimulus axis.

Standardized slope. The strategy of transforming slope estimates to a common, function-independent measure has been introduced by Strasburger (2001a), using the principle of calculating the slope of each function at its inflection point, which is the location of maximum slope. The required transformations are obtained by differentiating the psychometric function equation and evaluating the derivative at the inflection point, which is the location on the abscissa corresponding to the threshold parameter value for all except the Quick function. Table 2 lists the functions discussed previously, with expressions for their derivatives and for evaluation of the derivative at threshold.

The expressions in the last column of Table 2 deliver the local gradient of each function at its threshold. Hence, apart from the Quick function, these expressions may be used to calculate inflection point gradient $\beta^{\prime}$. The inflection point gradient provides a standard, function-independent measure of psychometric steepness, which may be interpreted unambiguously as indicating the increase in proportion of positive responses per log unit increase in stimulus level
(Strasburger, 2001a). This measure, in effect, matches functions at their inflection points. Strasburger (2001a) provides expressions, equivalent to those in Table 2 here, for calculating inflection point gradients of Gaussian, logistic, and Weibull functions. For the Quick function, in which the threshold parameter does not define the inflection point, Strasburger (2001a) also indicates that it may be rendered equivalent to the Weibull, using the threshold transformation discussed previously (Equations 15 and 16).

Function-to-function slope transformation. A second strategy for resolving slope differences across models is to transform the slope value from its originating psychometric function into some target function, rather than into a standardized slope representation. Although this approach does not bring different functions together with a common slope value, it does preserve original threshold values and delivers transformed slope values that may be interpreted as though estimated directly from data.

Function-to-function transformation adopts the principle of matching the local gradients of functions at the threshold, which generally produces close correspondence between functions in the central part of the curve extending above and below threshold. The poorest match between the functions then naturally occurs toward the tails. This approach may be described mathematically in terms of matching Taylor series expansions about the threshold. When applied to two-parameter models, the method reduces to one of matching tangents at the threshold. In this way, the basic structure of the psychometric function in the neighborhood of threshold is captured, and the peripheral detail is ignored as being of secondary importance. The calculations for these transformations involve evaluating the derivative of each psychometric function at its threshold location and are, therefore, essentially the same as those involved in the maximum slope measure above. The expressions in the last column of Table 2 provide the basis for all desired function-to-function transformations. To match the threshold gradients of two different functions, it is necessary only to set one of these expressions equal to another, then to rearrange the equation in terms of the desired parameter. For example, to transform slope

Table 1

Summary of Psychometric Functions, With Notation for Slope and Threshold Parameters

\begin{tabular}{llllcc}
\hline $\begin{array}{c}\text { Function } \\
\text { Designation }\end{array}$ & $\begin{array}{c}\text { Function } \\
\text { Type }\end{array}$ & $\begin{array}{c}\text { Kernel } \\
\text { Type }\end{array}$ & $\begin{array}{c}\text { Stimulus } \\
\text { Scale }\end{array}$ & $\begin{array}{c}\text { Slope } \\
\text { Parameter }\end{array}$ & $\begin{array}{c}\text { Threshold } \\
\text { Parameter }\end{array}$ \\
\hline G1 $x$ & Gaussian & difference (1) & $x$ & $\alpha$ & $\theta$ \\
G2 $\log x$ & Gaussian & log-ratio (2B) & $\log x$ & $\beta$ & $\log \theta$ \\
L1 $x$ & Logistic & difference (1) & $x$ & $\delta$ & $\theta$ \\
L2 $x$ & Logistic & ratio (2A) & $x$ & $\varepsilon$ & $\theta$ \\
L2 $\log x$ & Logistic & $\log$-ratio (2B) & $\log x$ & $\phi$ & $\log \theta$ \\
Q2 $x$ & Quick & ratio (2A) & $x$ & $\omega$ & $\theta$ \\
Q2 $\log x$ & Quick & $\log$-ratio (2B) & $\log x$ & $\omega$ & $\log \theta$ \\
W2 $x$ & Weibull & ratio (2A) & $x$ & $\omega$ & $\eta$ \\
W2 $\log x$ & Weibull & $\log$-ratio (2B) & $\log x$ & $\omega$ & $\log \eta$ \\
\hline
\end{tabular}

Note-Each function designation in column 1 reflects function type, kernel type, and stimulus scale, given in columns $2-4$. 
Table 2

Psychometric Function Equations, Derivatives, and Expressions for Slope Parameter Evaluation

\begin{tabular}{|c|c|c|c|}
\hline Function & Response Probability & Derivative of Function & $\begin{array}{c}\text { Derivative at } \\
x=\text { Threshold }\end{array}$ \\
\hline$P_{\mathrm{G} 1 x}(\theta, \alpha)$ & $\frac{\alpha}{\sqrt{2 \pi}} \int_{-\infty}^{x} \exp \left[\frac{-1}{2}\{\alpha(t-\theta)\}^{2}\right] d t$ & $\frac{\alpha}{\sqrt{2 \pi}} \exp \left[\frac{-\{\alpha(x-\theta)\}^{2}}{2}\right]$ & $\frac{\alpha}{\sqrt{2 \pi}}$ \\
\hline$P_{\mathrm{G} 2 \log x}(\theta, \beta)$ & $\frac{\beta}{\sqrt{2 \pi} \ln b} \int_{0}^{x} \frac{1}{t} \exp \left[\frac{-1}{2}\left\{\log _{b}\left[\frac{t}{\theta}\right]^{\beta}\right\}^{2}\right] d$ & $\frac{\beta}{\sqrt{2 \pi} \ln b} \frac{1}{x} \exp \left[-\frac{1}{2}\left\{\log _{b}\left[\frac{x}{\theta}\right]^{\beta}\right\}^{2}\right]$ & $\frac{1}{\sqrt{2 \pi} \ln b} \frac{\beta}{\theta}$ \\
\hline$P_{\mathrm{L} 1 x}(\theta, \delta)$ & {$[1+\exp \{\delta(\theta-x)\}]^{-1}$} & $\delta P_{\mathrm{L} 1 x}\left(1-P_{\mathrm{L} 1 x}\right)$ & $\frac{\delta}{4}$ \\
\hline$P_{\mathrm{L} 2 x}(\theta, \varepsilon)$ & {$\left[1+\left(\frac{\theta}{x}\right)^{\varepsilon}\right]^{-1}$} & $\frac{\varepsilon}{x} P_{\mathrm{L} 2 x}\left(1-P_{\mathrm{L} 2 x}\right)$ & $\frac{\varepsilon}{4 \theta}$ \\
\hline$P_{\mathrm{L} 2 \log x}(\theta, \phi)$ & {$\left[1+\exp \left\{\phi\left(\log _{b} \theta-\log _{b} x\right)\right\}\right]^{-1}$} & $\frac{1}{\ln b} \frac{\phi}{x} P_{\mathrm{L} 2 \log x}\left(1-P_{\mathrm{L} 2 \log x}\right)$ & $\frac{1}{4 \ln b} \frac{\phi}{\theta}$ \\
\hline$P_{\mathrm{Q} 2 x}(\theta, \omega)$ & $1-2^{--}$ & $(\ln 2) \frac{\omega}{\theta}\left(\frac{x}{\theta}\right)^{\omega-1}\left(1-P_{\mathrm{Q} 2 x}\right)$ & $\frac{\ln 2}{2} \frac{\omega}{\theta}$ \\
\hline$P_{\mathrm{Q} 2 \log x}(\theta, \omega)$ & $1-2^{\left[-b^{\left\{\omega\left(\log _{b} x-\log _{b} \theta\right)\right\}}\right]}$ & $\ln 2 \frac{\omega}{x} b^{\left\{\omega\left(\log _{b} x-\log _{b} \theta\right)\right\}}\left(1-P_{\mathrm{Q} 2 \log x}\right)$ & $\frac{\ln 2}{2} \frac{\omega}{\theta}$ \\
\hline$P_{\mathrm{W} 2 x}(\eta, \omega)$ & $1-e^{L}$ & $\frac{\omega}{\eta}\left(\frac{x}{\eta}\right)^{\omega-1}\left(1-P_{\mathrm{W} 2 x}\right)$ & $\frac{1}{e} \frac{\omega}{\eta}$ \\
\hline$P_{\mathrm{W} 2 \log x}(\eta, \omega)$ & $1-e^{\left[-b^{\left\{\omega\left(\log _{b} x-\log _{b} \eta\right)\right\}}\right]}$ & $\frac{\omega}{x} b^{\left\{\omega\left(\log _{b} x-\log _{b} \eta\right)\right\}}\left(1-P_{\mathrm{W} 2 \log x}\right)$ & $\frac{1}{e} \frac{\omega}{\eta}$ \\
\hline
\end{tabular}

parameter $\alpha$ of the Gaussian in $x(\mathrm{G} 1 x)$ to $\delta$ of the logistic in $x(\mathrm{~L} 1 x)$ involves solving the equation

$$
\frac{\alpha}{\sqrt{2 \pi}}=\frac{\delta}{4},
$$

from which we find

$$
\delta=\alpha \frac{4}{\sqrt{2 \pi}} .
$$

This same procedure may be followed for any desired transformation among the available combinations. Table 3 gives expressions for all slope conversions by application of this principle.

When using Table 3, select the originating function (i.e., the function to be converted) from the left-hand margin and the target function (to convert to) from the column headers; then read across the row of the originating function to the column of the target function, to obtain the required parametric expression for slope conversion. Table 3 includes slope transformations for the Quick function, but not for the Weibull. Earlier, it was noted that Weibull and Quick functions are equivalent when the difference between thresholds is removed, so conversion of the Weibull to other functions may be achieved by first transforming to a Quick function ( $\eta$ to $\theta$ ), then using Table 3 to transform the slope.

Table 3 specifies formulae for theoretical slope conversion from any originating function to any target function and is internally consistent for transitive multistage slope conversion. For example, if a logistic model such as L1x were to be fitted to experimental data and subsequently to be converted from $\mathrm{L} 1 x \rightarrow \mathrm{G} 2 \log x$ and, again, from $\mathrm{G} 2 \log x \rightarrow \mathrm{Q} 2 x$, the result would correspond exactly to that derived by converting directly from $\mathrm{L} 1 x \rightarrow \mathrm{Q} 2 x$. This transitivity property also holds for conversions involving the Weibull function, provided the appropriate threshold transformation is applied.

Slope expressed as stimulus spread. Just as threshold is expressed as a stimulus value corresponding to a particular response probability, so it is possible to express slope in terms of the stimulus range covered by some nonasymptotic portion of the function. This range, which may be called the spread (Treutwein \& Strasburger, 1999) or support (García-Pérez, 1998, 2001) of the function, will vary inversely with the value of the slope parameter. Spread may be determined by choosing two response 
Table 3

Expressions for Conversion of Slope Parameter Values Between Psychometric Function Models

\begin{tabular}{|c|c|c|c|c|c|c|}
\hline & $P_{\mathrm{G} 1 x}(\theta, \alpha)$ & $P_{\mathrm{G} 2 \log x}(\theta, \beta)$ & $P_{\mathrm{L} 1 x}(\theta, \delta)$ & $P_{\mathrm{L} 2 x}(\theta, \varepsilon)$ & $P_{\mathrm{L} 2 \log x}(\theta, \phi)$ & $P_{\mathrm{Q} 2 x}(\theta, \omega)$ \\
\hline$P_{\mathrm{G} 1 x}(\theta, \alpha)$ & & $\alpha \theta \ln b$ & $\alpha \frac{4}{\sqrt{2 \pi}}$ & $\alpha \theta \frac{4}{\sqrt{2 \pi}}$ & $\alpha \theta \ln b \frac{4}{\sqrt{2 \pi}}$ & $\alpha \frac{\theta}{\ln 2} \sqrt{\frac{2}{\pi}}$ \\
\hline$P_{\mathrm{G} 2 \log x}(\theta, \beta)$ & $\beta \frac{1}{\theta \ln b}$ & & $\beta \frac{1}{\theta \ln b} \frac{4}{\sqrt{2 \pi}}$ & $\beta \frac{1}{\ln b} \frac{4}{\sqrt{2 \pi}}$ & $\beta \frac{4}{\sqrt{2 \pi}}$ & $\beta \frac{1}{\ln 2 \ln b} \sqrt{\frac{2}{\pi}}$ \\
\hline$P_{\mathrm{L} 1 x}(\theta, \delta)$ & $\delta \frac{\sqrt{2 \pi}}{4}$ & $\delta \theta \ln b \frac{\sqrt{2 \pi}}{4}$ & & $\delta \theta$ & $\delta \theta \ln b$ & $\delta \theta \frac{1}{\ln 4}$ \\
\hline$P_{\mathrm{L} 2 x}(\theta, \varepsilon)$ & $\varepsilon \frac{1}{\theta} \frac{\sqrt{2 \pi}}{4}$ & $\varepsilon \ln b \frac{\sqrt{2 \pi}}{4}$ & $\varepsilon \frac{1}{\theta}$ & & $\varepsilon \ln b$ & $\varepsilon \frac{1}{\ln 4}$ \\
\hline$P_{\mathrm{L} 2 \log x}(\theta, \phi)$ & $\phi \frac{1}{\theta \ln b} \frac{\sqrt{2 \pi}}{4}$ & $\phi \frac{\sqrt{2 \pi}}{4}$ & $\phi \frac{1}{\theta \ln b}$ & $\phi \frac{1}{\ln b}$ & & $\phi \frac{1}{\ln 4 \ln b}$ \\
\hline$P_{\mathrm{Q} 2 x}(\theta, \omega)$ & $\omega \frac{\ln 2}{\theta} \sqrt{\frac{\pi}{2}}$ & $\omega \ln 2 \ln b \sqrt{\frac{\pi}{2}}$ & $\omega \frac{\ln 4}{\theta}$ & $\omega \ln 4$ & $\omega \ln 4 \ln b$ & \\
\hline
\end{tabular}

Note-Select an originating function (to be converted) from the list in column 1 and read across that row for expressions with which to convert to the target functions denoted in the headings of columns $2-7$.

probabilities and using the inverse of the psychometric function to calculate the stimulus values corresponding to these probabilities. The response probabilities define the support interval, and the difference between the corresponding stimulus values is the spread of the function. This approach has two clear advantages over that of using function parameters: It provides a common metric for expressing the effect of psychometric function slope that is mostly independent of the function model, and it expresses the effect of the slope in psychologically meaningful stimulus units.

In practice, any pair of response probabilities within the nonasymptotic region may be used to define spread in a standard manner, but two possibilities naturally predominate. For one, the quartile probabilities, .25 and .75, are used, so that the spread value defines the interquartile range (Chauhan et al., 1993; Strasburger, 2001a). The alternative, taking advantage of the fact that most psychometric functions are antisymmetric about the median threshold, is to define the deviation between threshold probability and some other level, either above or below threshold. Perhaps the most intuitive approach here is to select the range that is consistent with the conventional definition of spread in the Gaussian function - that is, the standard deviation. This is the range given by the difference between the stimulus value at response $P=.8413$ and that at the median threshold $(P=.5000)$. Table 4 provides expressions that may be used for the calculation of spread and deviation.

Note that the spread of a function between any desired response probabilities may be calculated using Table 4, column 2, which provides expressions for the inverse of each psychometric function, with the exception of the Gaussian, which has no closed-form solution for the inverse function. However, numerical approximations exist for the inverse cumulative Gaussian, one of which (Acklam, 2004; see the Appendix) is simple and accurate enough for all practical purposes. If measures of deviation are required, Table 4, column 3, provides general expressions for the deviation between threshold and any desired suprathreshold probability, and column 4 provides simplified expressions for a Gaussian equivalent deviation $\left(\sigma_{\mathrm{GED}}\right)$, which has the obvious advantage with Gaussian functions that it requires only the reciprocal of the slope to be calculated. Note that deviation expressions for functions in $x$ having ratio kernels (namely, L2x and Q $2 x$ ) include threshold values, since threshold and slope parameters in these cases do not exhibit algebraic independence.

Table 4 does not include expressions for Weibull functions $\mathrm{W} 2 x$ and $\mathrm{W} 2 \log x$, because threshold $\eta$ is located at $P(\eta)=.6321$, so any measure of deviation from threshold will not be directly comparable with other functions that use the median threshold parameter $\theta$. If slope is to be expressed in terms of a threshold-related deviation, use of the Quick function in place of the Weibull is advocated. Nevertheless, some caution is required when Table 4 is used to fit the Quick model. Due to the asymmetry of the Quick function, equal probability differences above and below threshold will give different deviation values. For this reason, deviation here has been uniquely defined only in relation to probabilities above threshold, assuming that this is likely to be the range of interest in practice.

One general limitation of expressing slope in terms of stimulus spread is that excessively wide support intervals will amplify the effect of the slope parameter, in that small changes in slope may correspond to relatively large changes in spread. It is therefore desirable that researchers adopt a standard approach and use a support interval 
Table 4

Inverse Functions and Expressions for Deviations

\begin{tabular}{|c|c|c|c|}
\hline Function & Stimulus $x$ at Response $P(x)$ & Suprathreshold Deviation $x-\theta$ & $\begin{array}{c}\text { Gaussian Equivalent Deviation } \\
\sigma_{\mathrm{GED}}=x_{0.8413}-\theta\end{array}$ \\
\hline$P_{\mathrm{G} 1 x}(\theta, \alpha)$ & $\begin{array}{l}\text { by numerical approximation } \\
\text { (see Appendix) }\end{array}$ & $\begin{array}{l}\text { by numerical approximation } \\
\text { (see Appendix) }\end{array}$ & $\frac{1}{\alpha}$ \\
\hline$P_{\mathrm{L} 1 x}(\theta, \delta)$ & $\theta+\frac{1}{\delta} \ln \left[\frac{P(x)}{1-P(x)}\right]$ & $\frac{1}{\delta} \ln \left[\frac{P(x)}{1-P(x)}\right]$ & $\frac{1.668}{\delta}$ \\
\hline$P_{\mathrm{L} 2 x}(\theta, \varepsilon)$ & $\theta\left[\frac{P(x)}{1-P(x)}\right]^{\frac{1}{\varepsilon}}$ & $\theta\left\{\left[\frac{P(x)}{1-P(x)}\right]^{\frac{1}{\varepsilon}}-1\right\}$ & $\theta\left\{5.301^{\frac{1}{\varepsilon}}-1\right\}$ \\
\hline$P_{\mathrm{Q} 2 x}(\theta, \omega)$ & $\theta\left\{\frac{-\ln [1-P(x)]}{\ln 2}\right\}^{\frac{1}{\omega}}$ & $\theta\left\{\left(\frac{-\ln [1-P(x)]}{\ln 2}\right)^{\frac{1}{\omega}}-1\right\}$ & $\theta\left\{2.656^{\frac{1}{\omega}}-1\right\}$ \\
\hline & Stimulus $\log _{b} x$ at Response $P\left(\log _{b} x\right)$ & $\begin{array}{c}\text { Suprathreshold Deviation } \\
\qquad \log _{b} x-\log _{b} \theta\end{array}$ & $\begin{array}{c}\text { Gaussian Equivalent Deviation } \\
\sigma_{\mathrm{GED}}=\log _{b} x_{0.8413}-\log _{b} \theta\end{array}$ \\
\hline$P_{\mathrm{G} 2 \log x}(\theta, \beta)$ & $\begin{array}{l}\text { by numerical approximation } \\
\text { (see Appendix) }\end{array}$ & $\begin{array}{l}\text { by numerical approximation } \\
\text { (see Appendix) }\end{array}$ & $\frac{1}{\beta}$ \\
\hline$P_{\mathrm{L} 2 \log x}(\theta, \phi)$ & $\log _{b} \theta+\frac{1}{\phi} \ln \left[\frac{P(x)}{1-P(x)}\right]$ & $\frac{1}{\phi} \ln \left[\frac{P(x)}{1-P(x)}\right]$ & $\frac{1.668}{\phi}$ \\
\hline$P_{\mathrm{Q} 2 \log x}(\theta, \omega)$ & $\log _{b} \theta+\frac{1}{\omega \ln b} \ln \left\{\frac{-\ln [1-P(x)]}{\ln 2}\right.$ & $\frac{1}{\omega \ln b}\left\{\ln \left(\frac{-\ln [1-P(x)]}{\ln 2}\right)\right\}$ & $\frac{0.977}{\omega \ln b}$ \\
\hline
\end{tabular}

that is relatively narrow. This is another argument in favor of expressing spread in terms of a moderately sized, onesided deviation criterion such as Gaussian equivalent deviation $\left(\sigma_{\mathrm{GED}}\right)$.

Figure 4 illustrates relationships between Gaussian equivalent deviation $\sigma_{\mathrm{GED}}$ and standard deviation $\sigma$, in different psychometric functions, using both arithmetic and logarithmic stimulus scales (see Figures 4A and 4B, respectively).

Figure 4 is constructed by using Table 3 to match the slopes of different psychometric functions at their median threshold $\theta$, then calculating $\sigma_{\mathrm{GED}}$ for a range of slope values, using the expressions in Table 4. Gaussian functions G1 $x$ and $\mathrm{G} 2 \log x$, in which $\sigma_{\mathrm{GED}}=\sigma$, provide (dashed) reference lines in Figures 4A and 4B, respectively. In each plot, there is close correspondence between $\sigma_{\mathrm{GED}}$ values of different psychometric functions when $\sigma$ is small (steep slopes) but poorer correspondence as $\sigma$ increases (shallow slopes), indicating how the goodness of match between functions deteriorates as slope decreases.

Figure 4A shows $\sigma_{\mathrm{GED}}$ plots for functions in $x$ matched at threshold value $\theta=1$. Note that the deviation exhibited by $\mathrm{L} 2 x$ and $\mathrm{Q} 2 x$ will vary with $\theta$, since slope varies with the value of the threshold parameter $\theta$ in these ratio kernel functions. The effect of threshold variation on the deviation of these functions will be significant, with both $\mathrm{L} 2 x$ and Q2 $x$ showing their nearest approach to G1 $x$ when $\theta$ is high but diverging markedly from G1 $x$ for values of $\theta<1$.
On the other hand, slopes (and deviations) of Gaussian and logistic functions G1 $x$ and L $1 x$ are unaffected by change in the value of the threshold parameter. This difference in behavior indicates that G1x and L $1 x$ are the preferred psychometric functions for arithmetic stimulus scales. If the Gaussian itself is to be avoided for mathematical reasons, logistic L1 $x$ remains as the function of choice.

For consistency, Figure 4B shows $\sigma_{\mathrm{GED}}$ plots for functions in $\log _{e} x$ matched at threshold value $\log _{e} \theta=0$. In this case, slopes (and hence, deviations) of all functions are unaffected by changes in threshold value.

\section{Discussion}

We have presented mathematical expressions through which differences in the slopes of psychometric functions may be resolved using three possible strategies. Attempts to match different psychometric functions in terms of slope are necessarily approximate, because functions of different mathematical forms have intrinsically different shapes, and each of the strategies considered has advantages and limitations. The most generally applicable approach is that of direct function-to-function slope transformation, which achieves an exact match between functions at the threshold location, using the natural parameter values of each function. In addition, consideration of function-to-function transformations (see Table 3) reveals a number of interesting features of the relationships between psychometric function models. 

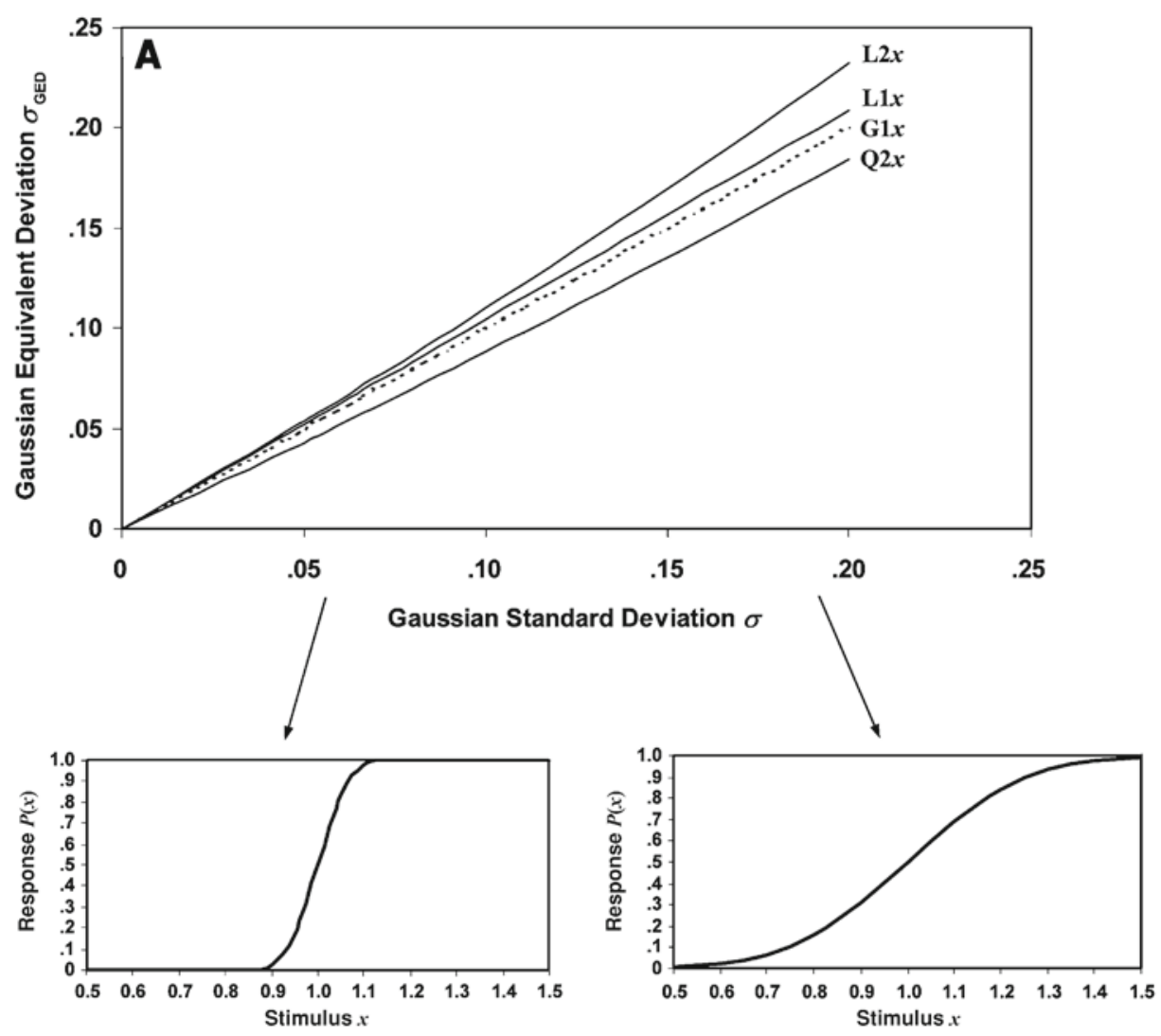

Figure 4. Relationship between Gaussian equivalent deviation $\sigma_{\text {GED }}$ and Gaussian standard deviation $\sigma$ for different psychometric function models matched at the median threshold. The Quick function always exhibits smaller deviation than does the Gaussian function, reflecting the steeper shape of the Quick/Weibull model in the region just above median threshold. All other functions are closely matched when $\sigma$ is small (steep slope; see the inset), and closeness of match deteriorates as $\sigma$ increases. (A) Functions for arithmetic stimulus scales.

Gaussian to logistic. An approach to relating Gaussian and logistic slope values in terms of deviation has been outlined elsewhere (Green, Richards, \& Forrest, 1989). The principle is that, with a Gaussian distribution, $\mu-\sigma$ (where $\mu=$ mean and $\sigma=$ standard deviation) corresponds to a response probability of .1587 (.5000 $.3413)$ and $\mu+\sigma$ corresponds to a response probability of .8413 $(.5000+.3413)$. Green et al. suggested that obtaining the same variation from the logistic function requires $\delta=1.65 \alpha$. Evaluation of the relevant expressions in Table 3 permits exact conversions.

For arithmetic scales,

$\mathrm{L} 1 x \leftarrow \mathrm{G} 1 x, \quad \delta=1.596 \alpha \quad$ (three decimal places), and for logarithmic scales,

$$
\mathrm{L} 2 \log x \leftarrow \mathrm{G} 2 \log x, \quad \phi=1.596 \beta \quad \begin{gathered}
\text { (three decimal } \\
\text { places). }
\end{gathered}
$$

Thus, there is mathematical consistency in the relationships between Gaussian and logistic functions on both arithmetic and logarithmic scales, and this relationship holds for any logarithmic base value.
Arithmetic to logarithmic scales. It has been pointed out that only ratio kernel forms of the psychometric function permit logarithmic transformation. Therefore, only two of our function types may be used for direct conversion between arithmetic and logarithmic scales: the Quick (Q2) and the logistic (L2). In each case, by using natural logarithms, identical functions can be obtained on both arithmetic and logarithmic scales with the same slope value:

$$
\text { Q2 } \log x \leftarrow \mathrm{Q} 2 x, \quad \omega=\omega,
$$

and

$$
\mathrm{L} 2 \log x \leftarrow \mathrm{L} 2 x, \quad \phi=\varepsilon .
$$

Note, however, that although the slope value of the Quick function remains constant when moving from an arithmetic to a logarithmic scale on any base $(b>1)$, the logistic slope value remains constant only with natural logarithms; that is, when $b \neq e$, then $\phi \neq \varepsilon$.

Of course, ratio kernel functions in $x$ do not exhibit algebraic independence of slope and threshold parameters. Therefore, to provide pairs of functions that are similar 


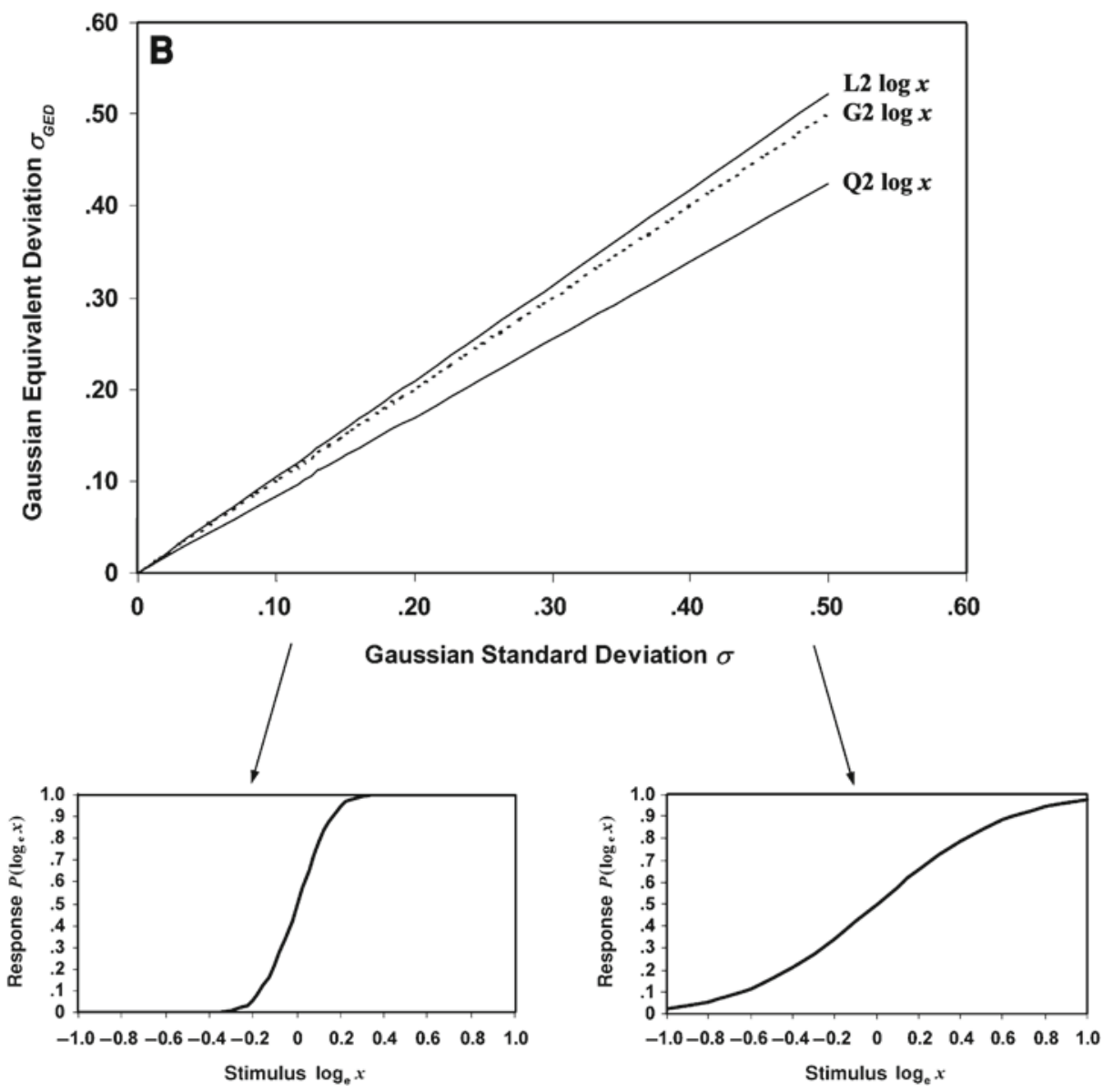

Figure 4 (Continued). (B) Functions for logarithmic stimulus scales.

in form and that give slope and threshold independence on both arithmetic and logarithmic scales, the natural choices are difference kernel and log-ratio kernel forms of the Gaussian and logistic functions (there is no difference kernel version of the Quick/Weibull function). Here, when natural logarithms are used to simplify relationships, it is found that for Gaussian functions,

$$
\mathrm{G} 2 \log x \leftarrow \mathrm{G} 1 x, \quad \beta=\alpha \theta,
$$

and similarly, for logistic functions,

$$
\mathrm{L} 2 \log x \leftarrow \mathrm{L} 1 x, \quad \phi=\delta \theta,
$$

so the slope on the logarithmic scale is equal to the slope on the arithmetic (linear) scale multiplied by the threshold value. This simple relationship between linear and $\log _{e}$ slope values has also been noted by Klein (2001).

Logarithmic stimulus scales. Logarithmic scaling of stimulus values is very popular in experimental and clinical psychophysics. For example, visual detection studies commonly plot functions with log luminance, amplitude, or contrast on the abscissa, whereas clinical measures of visual acuity use log minimum angle of resolution (logMAR), and instruments for visual field assessment use a decibel $\left(\log _{10}\right)$ scale of stimulus intensity. The popularity of logarithmic stimulus scales implies that psychometric functions fitted in practice will very often be of the log-ratio form. Thus, it is interesting to examine the relationships between the slope parameters of models Q2 $\log x, \mathrm{G} 2 \log x$, and L2 $\log x$. In each case, it is necessary to make a decision about which base value should be used for logarithmic transformation of the stimulus. Note that, although Table 3 presents general expressions for logarithms in any base $(b>1)$, relationships between functions are simplified if natural (base $e$ ) logarithms are used.

Consequently, it is possible to derive the following simple expressions:

$$
\begin{array}{ll}
\mathrm{G} 2 \log x \leftarrow \mathrm{Q} 2 \log x, & \beta=0.869 \omega, \\
\mathrm{L} 2 \log x \leftarrow \mathrm{Q} 2 \log x, & \phi=1.386 \omega,
\end{array}
$$

and

$$
\text { L2 } \log x \leftarrow \mathrm{G} 2 \log x, \quad \phi=1.596 \beta .
$$

All the aspects discussed above indicate the advantages of using natural (base $e$ ) logarithms for stimulus scales (Klein, 2001). 
Weibull and Quick functions. The use, in psychophysics, of both Weibull $\left(1-e^{- \text {kernel}}\right)$ and Quick $(1-$

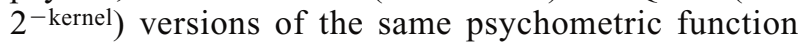
model is unfortunate. As was indicated previously, the Quick function is the only commonly used model in which the threshold parameter $\theta$ does not define the point of inflection. On the other hand, the Weibull is the only function in which the threshold parameter does not define the median (50\%) probability point. Therefore, all efforts to resolve slope differences by matching functions, either at the inflection point or at the median point, are frustrated by either the Quick or the Weibull function, respectively.

Thus, the expressions derived by Strasburger (2001a) for converting the slope parameter between Weibull and Gaussian/logistic functions are different from those presented above for converting between Quick and Gaussian/ logistic functions because, as was discussed previously, the Weibull matches at its inflection point (characteristic threshold $\eta$ ), whereas the Quick matches at the median threshold $\theta$. Using the Weibull in place of the Quick function gives

$$
\mathrm{G} 2 \log x \leftarrow \mathrm{W} 2 \log x, \quad \beta=0.922 \omega,
$$

and

$$
\mathrm{L} 2 \log x \leftarrow \mathrm{W} 2 \log x, \quad \phi=1.471 \omega .
$$

Note that the conversion factors for the Weibull are simply 1.061 times those for the Quick function. This may be demonstrated by comparing the slope expressions for the two functions given in Table 2. For the Weibull function,

$$
G_{\text {Weibull }}=\frac{d P}{d x_{\text {Weibull }}}=\frac{1}{e} \frac{\omega}{\eta},
$$

where $G_{\text {Weibull }}$ denotes the gradient of the Weibull function at its natural (characteristic) threshold. Similarly, for the Quick function,

$$
G_{\text {Quick }}=\frac{d P}{d x_{\text {Quick }}}=\frac{\ln 2}{2} \frac{\omega}{\theta},
$$

where now $G_{\text {Quick }}$ denotes the gradient of the Quick function at its natural (median) threshold. Now, since it has been acknowledged that the two functions have the same shape despite the difference between thresholds, the threshold parameters may be disregarded. This is equivalent to treating the expressions as though each function is plotted on a stimulus scale normalized to its own threshold value; hence, each threshold takes a value of 1 (Klein, 2001; Strasburger, 2001a). Subsequently, rearranging the equations above gives

$$
\omega=e G_{\text {Weibull }}
$$

and

$$
\omega=\frac{2}{\ln 2} G_{\text {Quick }}
$$

hence,

$$
\begin{aligned}
G_{\text {Weibull }} & =\frac{2}{e \ln 2} G_{\text {Quick }} \\
& =1.061 G_{\text {Quick }} \quad \text { (three decimal places). }
\end{aligned}
$$

Thus, it may be seen that, when Weibull and Quick functions are superimposed, the gradient at $\eta$ is 1.061 times greater than the gradient at $\theta$.

In practice, therefore, it is important to be explicit about whether the Weibull or the Quick formulation is being used. Indeed, it would make matters easier, when a function of Weibull shape is required, if researchers would adopt either the Weibull or the Quick formulation, but not both. In this case, we advocate using the Quick formulation, precisely because its threshold lies naturally at the median point of the probability range, in keeping with all other forms of the psychometric function under consideration. This enables slope conversion to be applied consistently to all psychometric functions without requiring any modification of the threshold parameter.

\section{Summary}

1. Psychometric functions, relating response probability to stimulus intensity, are most commonly described using one of three mathematical models: Gaussian, logistic, or Weibull. All three models produce similar sigmoidshaped functions that may be specified by two parameters: one determining (principally) the threshold location on the stimulus scale and another determining (principally) the slope of the function at the threshold location.

2. Two kernel forms have been identified, which appear in popular models of the psychometric function. The difference kernel is not amenable to logarithmic transformation and so may be used only on arithmetic stimulus scales. The ratio kernel may be used on both arithmetic and logarithmic stimulus scales, as appropriate.

3. For conversion of stimulus values between arithmetic and logarithmic scales, ratio kernel forms of the psychometric function have an advantage in that they deliver identical response probabilities (however, see Point 4 below).

4. In order to ensure algebraic independence of threshold and slope parameters, (i.e., plotted functions appear parallel when only the threshold value changes), it is necessary to adopt the difference kernel on arithmetic stimulus scales and the log-ratio kernel on logarithmic scales.

5. Differences between the slope parameters of fitted functions may be resolved by using Table 2 to calculate a function-independent standardized value (Strasburger, 2001a). For all except the Quick function, this transformation will give the maximum slope of the function - that is, the gradient at the inflection point.

6 . Conversion of slope parameters between psychometric function models, retaining natural slope and threshold parameter values, may be achieved consistently by matching functions at the median threshold $P(\theta)=.50$, using the formulae in Table 3. In order to accommodate the general Weibull model using this principle, it is desirable to adopt the Quick $\left(1-2^{- \text {kernel})}\right.$ formulation in which threshold is naturally located at the $50 \%$ probability value. Researchers who prefer to adopt the Weibull $\left(1-e^{- \text {kernel }}\right)$ formulation for function fitting and who wish to compare slopes with Gaussian or logistic data fits could first convert to the Quick formulation by transformation from character- 
istic threshold $\eta$ to median threshold $\theta$; then Table 3 could be used to convert between slope parameters.

7. Slope conversions between arithmetic and logarithmic stimulus scales and between different functions using logarithmic stimulus scales are simplified if natural (base e) logarithms are used.

8. Functions having different slopes may also be compared in terms of their spread on the stimulus axis between any two designated response probabilities. This provides a function-independent measure that is inversely related to function slope and is expressed in stimulus units. It is convenient to choose probabilities that correspond to the Gaussian standard deviation $(\sigma)$, so that the spread of any function may be expressed in terms of its Gaussian equivalent deviation $\left(\sigma_{\mathrm{GED}}\right)$.

9. Finally, of all the commonly used psychometric function models, the logistic has a number of distinct advantages for practical application.

9.1. Logistic functions offer ease of mathematical analysis and a structure that is antisymmetric about the median (50\%) threshold location.

9.2. Logistic functions for use on arithmetic and logarithmic scales (namely, L1 $x$ and L2 $\log x$ ) exhibit the same kernel structure, and there is a simple relationship between their slope parameter values when natural logarithms are used.

9.3. Expressions for calculation of spread or deviation $\left(\sigma_{\mathrm{GED}}\right)$ are identical for logistic functions on arithmetic and logarithmic scales (namely, L1 $x$ and L2 $\log x$ ).

9.4. Gaussian equivalent deviation $\left(\sigma_{\mathrm{GED}}\right)$ values for $\mathrm{L} 1 x$ and L2 $\log x$ correspond closely to standard deviations $(\sigma)$ for G1x and G2 $\log x$ and are unaffected by changes in the threshold parameter value.

\section{REFERENCES}

ACKLAM, P. J. (2004). An algorithm for computing the inverse normal cumulative distribution function. Retrieved November 12, 2004, from http://home.online.no/ pjacklam/notes/invnorm.

BERKSON, J. (1951). Why I prefer logits to probits. Biometrics, 7, 327 339.

BlackWEll, H. R. (1953). Studies of the form of visual threshold data. Journal of the Optical Society of America, 43, 456-463.

Chauhan, B. C., Tompkins, J. D., LeBlanc, R. P., \& McCormick, T. A. (1993). Characteristics of frequency-of-seeing curves in normal subjects, patients with suspected glaucoma, and patients with glaucoma. Investigative Ophthalmology \& Visual Science, 34, 3534-3540.

Evans, M., Hastings, N., \& Peacock, B. (1993). Statistical distributions (2nd ed.). New York: Wiley.

García-Pérez, M. A. (1998). Forced-choice staircases with fixed step sizes: Asymptotic and small sample properties. Vision Research, 38, 1861-1881.

García-Pérez, M. A. (2001). Yes-no staircases with fixed step sizes: Psychometric properties and optimal setup. Optometry \& Vision Science, 78, 56-64.
Green, D. M., Richards, V. M., \& Forrest, T. G. (1989). Stimulus step size and heterogeneous stimulus conditions in adaptive psychophysics. Journal of the Acoustical Society of America, 86, 629-636.

Green, D. M., \& Swets, J. A. (1966). Signal detection theory and psychophysics. Los Altos, CA: Peninsula.

HARVEY, L. O., JR. (1986). Efficient estimation of sensory thresholds. Behavior Research Methods, Instruments, \& Computers, 18, 623632 .

KAERNBACH, C. (2001). Slope bias of psychometric functions derived from adaptive data. Perception \& Psychophysics, 63, 1389-1398.

KInG-Smith, P. E., \& Rose, D. (1997). Principles of an adaptive method for measuring the slope of the psychometric function. Vision Research, 37, 1595-1604.

KleIN, S. A. (2001). Measuring, estimating, and understanding the psychometric function: A commentary. Perception \& Psychophysics, 63, 1421-1455.

Kontsevich, L. L., \& Tyler, C. W. (1999). Bayesian adaptive estimation of psychometric slope and threshold. Vision Research, 39, 2729-2737.

Laming, D. (1986). Sensory analysis. London: Academic Press.

LIPETZ, L. E. (1969). The transfer functions of sensory intensity in the nervous system. Vision Research, 9, 1205-1234.

Macmillan, N. A., \& Creelman, C. D. (1991). Detection theory: A user's guide. Cambridge: Cambridge University Press.

Nachmias, J. (1981). On the psychometric function for contrast detection. Vision Research, 21, 215-223.

NaKa, K. I., \& Rushton, W. A. H. (1966). S-potentials from colour units in the retina of fish (Cyprinidae). Journal of Physiology, 185, 536-555.

Patterson, V. H., Foster, D. H., \& Heron, J. R. (1980). Variability of visual threshold in multiple sclerosis: Effect of background luminance on frequency of seeing. Brain, 103, 139-147.

Pelli, D. G. (1987). On the relation between summation and facilitation. Vision Research, 27, 119-123.

QUICK, R. F. (1974). A vector magnitude model for contrast detection. Kybernetik, 16, 65-67.

Snoeren, P. R., \& Puts, M. J. H. (1997). Multiple parameter estimation in an adaptive psychometric method: MUEST, an extension of the QUEST method. Journal of Mathematical Psychology, 41, 431439.

Strasburger, H. (2001a). Converting between measures of slope of the psychometric function. Perception \& Psychophysics, 63, 1348-1355.

STRASBURGER, H. (2001b). Invariance of the psychometric function for character recognition across the visual field. Perception \& Psychophysics, 63, 1356-1376.

Treutwein, B. (1995). Adaptive psychophysical procedures. Vision Research, 35, 2503-2522.

Treutwein, B., \& Strasburger, H. (1999). Fitting the psychometric function. Perception \& Psychophysics, 61, 87-106.

Tyler, C. W. (1997). Why we need to pay attention to psychometric function slopes. OSA Technical Digest Series, 1, 29-32.

Watson, A. B. (1979). Probability summation over time. Vision Research, 19, 515-522.

Watson, A. B., \& Pelli, D. G. (1983). QUEST: A Bayesian adaptive psychometric method. Perception \& Psychophysics, 33, 113-120.

WEIBULL, W. (1951). A statistical distribution function of wide applicability. Journal of Applied Mechanics, 18, 292-297.

Wichmann, F. A., \& Hill, N. J. (2001). The psychometric function: I. Fitting, sampling, and goodness of fit. Perception \& Psychophysics, 63, 1293-1313.

WiLSON, H. R. (1980). A transducer function for threshold and suprathreshold human vision. Biological Cybernetics, 38, 171-178. 


\section{APPENDIX}

An Algorithm for Computing the Inverse Cumulative Normal Distribution Function (Acklam, 2004)

Define the following constants:

$a 1=-3.969683028665376 \mathrm{e}+01$

$a 2=2.209460984245205 \mathrm{e}+02$

$a 3=-2.759285104469687 \mathrm{e}+02$

$a 4=1.383577518672690 \mathrm{e}+02$

$a 5=-3.066479806614716 \mathrm{e}+01$

$a 6=2.506628277459239 \mathrm{e}+00$

$b 1=-5.447609879822406 \mathrm{e}+01$

$b 2=1.615858368580409 \mathrm{e}+02$

$b 3=-1.556989798598866 \mathrm{e}+02$

$b 4=6.680131188771972 \mathrm{e}+01$

$b 5=-1.328068155288572 \mathrm{e}+01$

Then, for any response probability $p$ between .0275 and .9750 , calculate the standard normal deviate $z$ :

$q=p-0.5$

$r=q \times q$

$z=\frac{(((((a 1 \times r+a 2) \times r+a 3) \times r+a 4) \times r+a 5) \times r+a 6) \times q}{(((((b 1 \times r+b 2) \times r+b 3) \times r+b 4) \times r+b 5) \times r+1)}$.

Hence, calculate stimulus level $x$ :

$x=\frac{z}{\text { slope }}+\theta$,

where slope is the psychometric function slope value and $\theta$ is the median threshold. 